\title{
Ultrasensitive Mode-Localized Micromechanical Electrometer
}

\author{
P. Thiruvenkatanathan, J. Yan, and A. A. Seshia*, \\ Department of Engineering, \\ University of Cambridge, \\ Cambridge, United Kingdom. \\ *E-mail: aas41@cam.ac.uk
}

\begin{abstract}
We report a highly sensitive prototype micromechanical electrometer that employs the phenomena of mode-localization and curve veering for monitoring minute charge fluctuations across an input capacitor. The device consists of a pair of weakly coupled, nearly identical single crystal silicon, double-ended tuning fork (DETF) resonators. An addition of charge across an input capacitor on one of the coupled resonators induces a differential axial strain on that resonator relative to the other consequently perturbing the structural symmetry of the nearly periodic system. The resulting shifts in the eigenstates for the same magnitudes of charge input are theoretically and experimentally demonstrated to be nearly three orders of magnitude greater than corresponding resonant frequency variations. The topology chosen may also be adapted for force or strain monitoring thereby widening the relevance of the results reported here to precision inertial sensing as well.
\end{abstract}

\section{INTRODUCTION}

Electrometers, devices that are used to measure precise quantities of charge, have been a focus of research for over a hundred years. A wide variety of concepts have been proposed and demonstrated for accurately monitoring charge fluctuations across an input capacitor [1 - 5]. At present, however, the cryogenically cooled single electron transistor (SET) electrometers that utilize the phenomenon of quantum tunneling for charge detection, are seen as the benchmark for high sensitivity charge sensing, with demonstrated resolutions down to $10^{-5} e / \sqrt{\mathrm{Hz}} \quad$ [2]. While these solid-state electrometers offer excellent charge resolutions, they suffer from the inherent disadvantages of limited operational bandwidth and their need for operation at sub-Kelvin temperatures (in order to reduce thermal noise effects [2]).

More recently, the mechanical detection of charge using micro- and nano-mechanical resonators has also been proposed as a highly sensitive approach for charge sensing [3, 6]. The output of these resonant mechanical electrometers relies on tracking relative shifts in the resonant frequency of a micro- / nano-mechanical structure for induced variations in charge across an input gate capacitor. They offer the twin benefits of high charge resolution and large dynamic range

This research is funded in part by the British Council (UKIERI), Churchill College and the Cambridge Commonwealth Trust (University of Cambridge, UK.) making them attractive alternative platforms for charge sensing applications [3]. Resolution in the sub-electron regime has been experimentally demonstrated using nanometer scale mechanical resonators at relatively larger temperatures (of about $4.2 \mathrm{~K}$ ) [3]. In what follows, we propose the use of an alternate mechanism of charge sensing in such micro- and nanomechanical resonators by using the phenomenon of vibration mode-localization and the associated curve veering effect. Besides the higher sensitivity, such mode-localized mechanical electrometers also offer the added advantage of intrinsic common mode rejection making them less susceptible to false positive outputs arising from ambient environmental fluctuations. Furthermore, the topology chosen in this work may also be adapted for force or strain monitoring thereby widening the relevance of the results reported here to precision inertial sensing as well. Sensitivities that are orders of magnitude greater than the more conventionally used resonant frequency shift approach are experimentally demonstrated.

\section{MODE LOCALIZATION AND CURVE VEERING}

It is well known that in an array of weakly coupled, nearly identical resonators, the presence of very small periodicitybreaking structural irregularities inhibits the long-range propagation of vibration, leading to a confinement of vibration energy to small geometric regions. The extent of this vibration energy confinement depends not only on the magnitude of the induced disorder, but also on the strength of internal coupling between the resonators with weaker coupling resulting in stronger localization of the vibration modes [7]. It is also known that weakly coupled, nearly identical resonators exhibit an abrupt divergence of the loci of their eigenvalues when these are plotted against a parameter representing the symmetry-breaking disorder in the system [8, 9]. This phenomenon, often referred to as 'eigenvalue curve veering' or simply 'curve veering', was first observed by Leissa in a pioneer paper [10]. The eigenfunctions associated with the eigenvalues on each locus swap trajectories during veering, resulting in strong mode localization under conditions of weak internal coupling between the resonators [ $8-10]$. It has been shown that the existence of close eigenvalues in a coupled 
vibratory system is likely to cause the occurrence of both curve veering and mode localization suggesting that they are both manifestations of the same drastic phenomenon $[8,11-$ 13].

In order to understand the underlying physics behind the phenomena, consider two resonators coupled through a weak coupling spring $\left(k_{c}\right)$ as represented in the discretized model shown in fig. 1 .

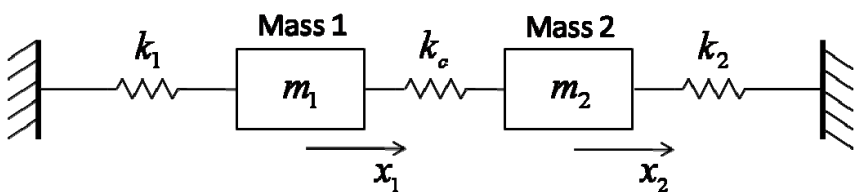

Figure 1: Lumped element model of a coupled two degree-of-freedom spring mass system.

In Fig. 1, ' $m$ ' represents the mass of resonators 1 and 2 $\left(m_{1}=m_{2}=m\right) ; \quad k_{1}$ and $k_{2}\left(=k_{1}+\Delta k\right)$ represent their respective stiffnesses; $k_{c}$ - the internal coupling spring constant; $x_{1}, x_{2}$ - their displacements. The two important parameters that we pay special attention to are the nondimensionalized coupling factor $\left(\kappa=k_{c} / k_{1}\right)$ between the two oscillators and the non-dimensionalized stiffness disorder on resonator 2 relative to resonator $1 \quad\left(\delta=\Delta k / k_{1}\right)$. The eigenvalue problem for undamped free vibration may be expressed as-

$$
\left|\begin{array}{cc}
\beta_{1}-\lambda & -\kappa \\
-\kappa & \beta_{2}-\lambda
\end{array}\right|=0
$$

where $\beta_{1}=1+\kappa ; \quad \beta_{2}=1+\kappa+\delta$ and $\lambda=\omega_{n}^{2} / k_{1} / m\left(\omega_{n}\right.$ represents the angular resonant frequencies of vibration for the two modes $n=1 ; 2)$. The corresponding eigenvalues may be estimated from (1) as -

$$
\lambda=\frac{1}{2}\left\{\beta_{1}+\beta_{2} \pm \sqrt{\left(\beta_{1}-\beta_{2}\right)^{2}+4 \kappa^{2}}\right\}
$$

with eigenvector components given by:

$$
\frac{x_{2}}{x_{1}}=\frac{1+\kappa-\lambda}{\kappa} .
$$

When the disorder in the stiffness of resonator 2 is zero, i.e., when $\delta=0 ;\left(\beta_{1}=\beta_{2}\right)$, the system is tuned or ordered and the eigenvalues and the corresponding eigenvectors of the system may be expressed as -

$$
\lambda_{01}=1, \frac{x_{2}}{x_{1}}=+1 ; \lambda_{02}=1+2 \kappa, \frac{x_{2}}{x_{1}}=-1
$$

Under conditions of weak internal coupling $(\kappa<<1)$, plotting the loci of the two eigenvalues of the system $(\lambda)$ versus the disorder in the stiffness of resonator $2(\delta)$ yields Fig. 2. It may be observed that the loci of the two eigenvalues do not cross but rather veer abruptly with high local curvatures as the two eigenvalues approach each other. The point of divergence corresponds to the point at which the system is symmetric $\left(\beta_{1}=\beta_{2}\right)$ as illustrated in Fig. 2 and represented in (4). At values where $\left(\beta_{1}-\beta_{2}\right)^{2}>>\left(4 \kappa^{2}\right)$, however, the modes localize as seen from (3) and (2) and illustrated in Fig. 2. The relative shifts in the magnitudes of the normalized eigenvectors (eigenstates) due to small perturbations in stiffness of one of the resonators may then be expressed as [7]

$$
\left|\frac{u_{n}-u_{0 n}}{u_{0 n}}\right| \cong \frac{\delta}{4 \kappa}=\left|\frac{\Delta k}{4 k_{c}}\right| ;(n=1,2) .
$$

where $u_{\mathrm{n}}$ and $u_{0 \mathrm{n}}$ represent the perturbed and unperturbed normalized two-component eigenvectors (or eigenstates) that are deduced from the amplitudes of vibration of each of the coupled resonators at the two fundamental modes ( $n$ denotes the fundamental mode of vibration).

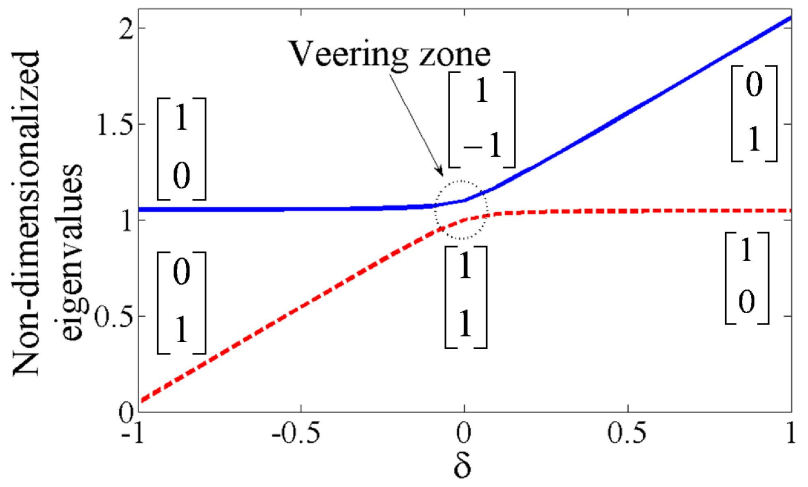

Figure 2: Loci of the dimensionless eigenvalues of the two coupled oscillators in terms of $\delta$.

Comparing this with the relative shift in the resonant frequency for the same induced perturbation in stiffness -

$$
\left|\frac{f-f_{0}}{f_{0}}\right| \cong\left|\frac{\Delta k}{2 k}\right|,
$$

it can be noticed that for any value of $k_{c}<k / 2$, the relative shifts in the eigenstates are greater than those in frequency. It is to be noted that in (2), $f_{0}$ and $f$ represent the frequency before and after inducing a stiffness perturbation and $k$ represents the unperturbed stiffness of the resonator platform $\left(k=k_{1}\right)$. Sensitivity enhancements exceeding three orders of magnitude relative to the more conventionally used resonant frequency shift approach have been reported using this sensing paradigm by exploiting this critical dependence of the eigenstate variations on the strength of internal coupling [7]. In this paper, unlike previous attempts, we incorporate this paradigm of mechanical sensing for monitoring minute charge fluctuations across an input capacitor. We also utilize the electrical spring softening effect to electrically tune the system to symmetry by plotting the eigenvalue loci of the system until they veer. We then use the point of veering to experimentally deduce the scaled coupling spring constant and utilize the value thus deduced, to accurately predict the relative shifts in the eigenstates due to induced charge variations.

\section{MODE-LOCALIZED CHARGE SENSING}

The concept is demonstrated using a pair of mechanically coupled nearly identical double ended tuning fork (DETF) resonators subjected to differential strain modulation. We 
utilize this particular topology as the topology may be directly adapted for force or strain monitoring thereby widening the relevance of the results reported here to precision inertial sensing applications as well. A schematic of the device is shown in Fig. 3.

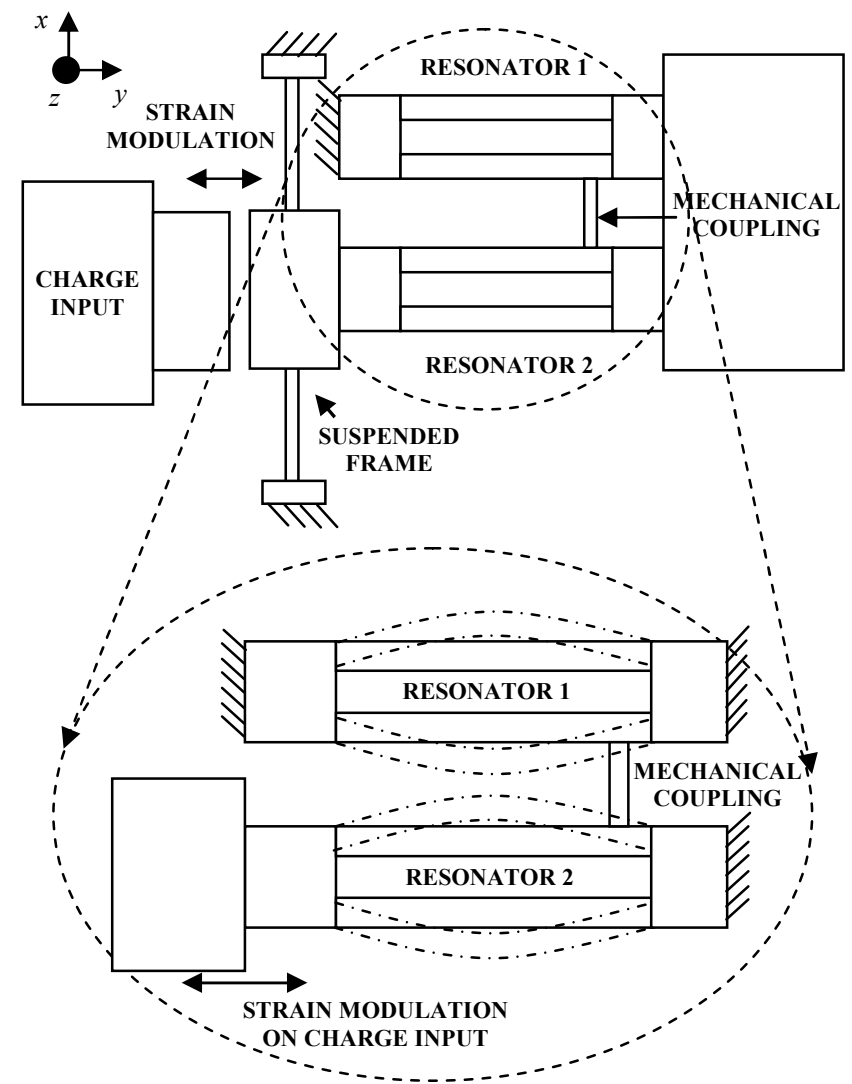

Figure 3: Schematic of mode-localized micromechanical electrometer

The charge sensing element consists of two DETF resonators coupled mechanically through the base. The resonators are actuated in the out-of-phase mode of vibration (as illustrated in the inset of Fig. 3) as this mode is associated with higher quality factor (Q) of resonance. One of the coupled tuning forks is anchored at both ends (resonator 1 in Fig. 3). The other resonator (resonator 2) however, has one end anchored with the other connected to a freely suspended frame constrained to move in the horizontal axis (y axis) as illustrated in Fig. 3. A charge actuation electrode is located adjacent to the suspended frame and forms the input of the electrometer. An introduction of charge $(q)$ through the charge input electrode causes an electrostatic force given by:

$$
F=\frac{q^{2}}{2 \varepsilon_{0} A_{c a p}}
$$

where $A_{\text {cap }}$ represents the area of the charge actuation capacitor. This electrostatic force in turn induces an axial strain on that resonator connected to the suspended frame while the other resonator undergoes no strain modulation, consequently breaking the initial periodicity of the system and inducing a localization of the vibration modes.

The spring constant of a doubly clamped beam of length $L$, subjected to an axial force $F$ may be written as [6]:

$$
k_{\text {axial }} \approx \frac{192 E I}{L^{3}}\left(1+\frac{L^{2} F}{40 E I}\right)
$$

where $E$ represents the Young's modulus of the elasticity and $I$, the second moment of area of the beam for in-plane lateral deflections. From equation (8), the shift in spring constant caused by the electrostatic force represented in equation (7) may be written as [6]:

$$
\Delta k=k_{\text {axial }}-k \approx \frac{192 E I}{L^{3}}\left(\frac{L^{2} F}{40 E I}\right)=\frac{4.8 F}{L}
$$

In the case of a double ended tuning fork that comprises of two doubly-clamped beams in parallel, the axial load gets distributed evenly between the beams and hence, the change in the stiffness of the DETF resonator subjected to strain modulation may be re-expressed from equations (9) and (7) as:

$$
\Delta k \approx \frac{2.4 F}{L}=\frac{1.2 q^{2}}{\varepsilon_{0} A_{c a p} L}
$$

Substituting equation (10) in equations (5) and (6) enables the evaluation of the relative shift in the eigenstates and those in resonant frequency for an induced charge addition of $q$ on the charge input electrode:

$$
\begin{aligned}
& \left|\frac{u_{n}-u_{0 n}}{u_{0 n}}\right| \approx \frac{\delta}{4 \kappa}=\frac{0.01875 q^{2} L^{2}}{\varepsilon_{0} A_{\text {cap }} W^{3} E H \kappa} \\
& \left|\frac{f-f_{0}}{f_{0}}\right| \approx \frac{0.0375 q^{2} L^{2}}{\varepsilon_{0} A_{\text {cap }} W^{3} E H}
\end{aligned}
$$

Comparing equations (11) and (12), it is clear that by simply weakening the strength of internal coupling between the two resonators, the relative shifts in the eigenstates may be as made greater than corresponding resonant frequency variations for the same charge input of $q$ across the charge input port. This implies that by simply reducing the strength of internal coupling, charge sensitivity enhancements as high as orders of magnitude greater than conventional resonant mechanical electrometers may be obtained.

It is to be noted that further enhancements in sensitivity may also be attained through the incorporation of force leverage mechanisms that are capable of amplifying the input electrostatic force before being communicated on to one of the coupled resonators. Such mechanical force leverage mechanisms have traditionally been employed in resonant inertial sensor systems [14] and have demonstrated force amplification factors as high as 80 [15]. 


\section{A. Device description and characterization}

The device reported here was fabricated in a commercial foundry process using the silicon-on-insulator microelectromechanical systems (SOI-MEMS) process through MEMSCAP Inc., USA. Each of the tines in both tuning forks was designed to be $25 \mu \mathrm{m}$ thick, $300 \mu \mathrm{m}$ long and $6 \mu \mathrm{m}$ wide with a gap of $6 \mu \mathrm{m}$ between the tines. Each of the resonators was driven and sensed using capacitive transduction. Actuation was achieved using parallel plates of equal dimensions, attached to either side as shown in the optical micrograph in Fig. 4.

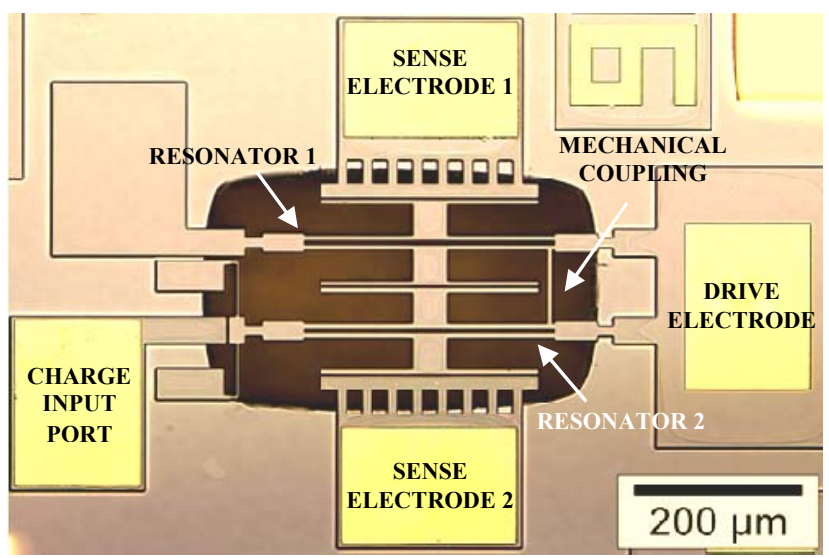

Figure 4: Optical micrograph of weakly coupled DETF resonators subjected to differential strain modulation.

A summary of the dimensions and design parameters is given in Table 1.

TABLE I. DEVICE DIMENSIONS

\begin{tabular}{|c|c|c|}
\hline \multicolumn{3}{|c|}{ Device dimensions and design parameters } \\
\hline Quantity & Symbol & Value \\
\hline Device thickness & $H$ & $25 \mu \mathrm{m}$ \\
\hline Tine length & $L$ & $300 \mu \mathrm{m}$ \\
\hline Tine width & $W$ & $6 \mu \mathrm{m}$ \\
\hline Electrode length & $l$ & $260 \mu \mathrm{m}$ \\
\hline Transduction gap & $G$ & $2 \mu \mathrm{m}$ \\
\hline Input charge electrode width & $w$ & $34 \mu \mathrm{m}$ \\
\hline Input charge actuation gap & $g$ & $2 \mu \mathrm{m}$ \\
\hline Scaled coupling factor & $\kappa$ & $3.52 \mathrm{e}-3$ \\
\hline
\end{tabular}

The fabricated devices were tested under vacuum $(\approx 50 \mathrm{mT}$ orr $)$ in a custom vacuum chamber. A schematic of the measurement setup is shown in Fig. 5. It may be observed from Fig. 5 that any disparity between the DC voltage on the charge input port and that on resonator 2 should result in a variation of the static component of the electrostatic attractive force in the charge actuation capacitor consequently inducing a differential axial strain within the nearly periodic system. This should in turn cause a localization of the vibration modes resulting in drastic variations in the modal dynamics. The resulting shifts in eigenstates deduced from the relative amplitudes of vibration of each of the coupled resonators at the eigenvalues (measured from their respective sense electrodes) yield the output of the electrometer.

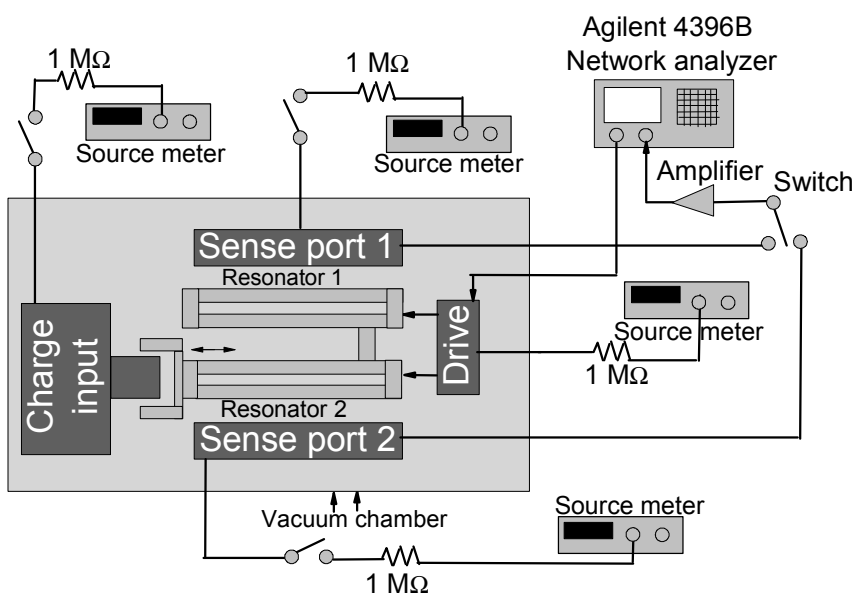

Figure 5: Schematic of experimental setup.

\section{B. Device calibration}

Initially, in order to deduce the unperturbed response of the system, the effective charge input (through the charge input port in Fig. 4) was maintained at $0 \mathrm{C}$ whilst driving both the resonators through the drive port. Equal magnitudes of DC voltages were applied on both the resonators to induce capacitive actuation and at the same time, avoid any periodicity breaking irregularity between the two coupled structures due to differential electrical spring softening within the system [7]. The eigenstates of the coupled responses were then deduced by simply measuring the relative S21 transmission responses arising from the motional currents at sense electrodes 1 and 2 respectively. The measured transmission frequency responses are illustrated in Fig. 6.

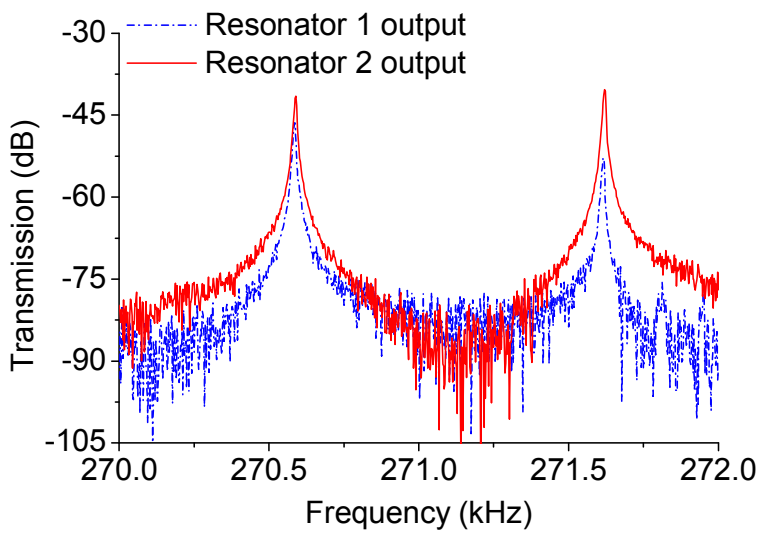

Figure 6: Unperturbed transmission responses of resonators 1 and 2 measured from their respective sense electrodes.

The measured responses reveal the presence of two resonant modes that are close in frequency indicating the existence of a weak elastic coupling element between the 
resonators. Before proceeding with illustrating the functionality of the device as a charge sensor, it becomes mandatory to experimentally quantify the strength of internal coupling between the tuning forks. This becomes especially important in mode-localized sensing as any enhancements in the sensitivity in such sensors, depend critically on the ability to predict the strength of the internal coupling spring constant as may be observed from (11). Hence, in order to obtain an experimental measure of the coupling spring constant, the loci of the eigenvalues of the vibratory system are plotted as a function of induced stiffness variations on one of the coupled resonators. This is achieved by first applying a DC bias voltage of $10 \mathrm{~V}$ on the drive electrode (refer Fig. 5) in addition to providing an $\mathrm{AC}$ input from the Vector network analyzer (VNA) corresponding to $-20 \mathrm{dBm}$. The DC voltage on the sense electrodes of each of the resonators are initially maintained at $0 \mathrm{~V}$. The DC bias voltage on sense electrode 2 is then tuned to alter the stiffness of resonator 2 maintaining that of resonator 1 constant. Plotting the variations in frequencies of the two modes as a function of the induced stiffness disorder on resonator 2 results in Fig. 7.

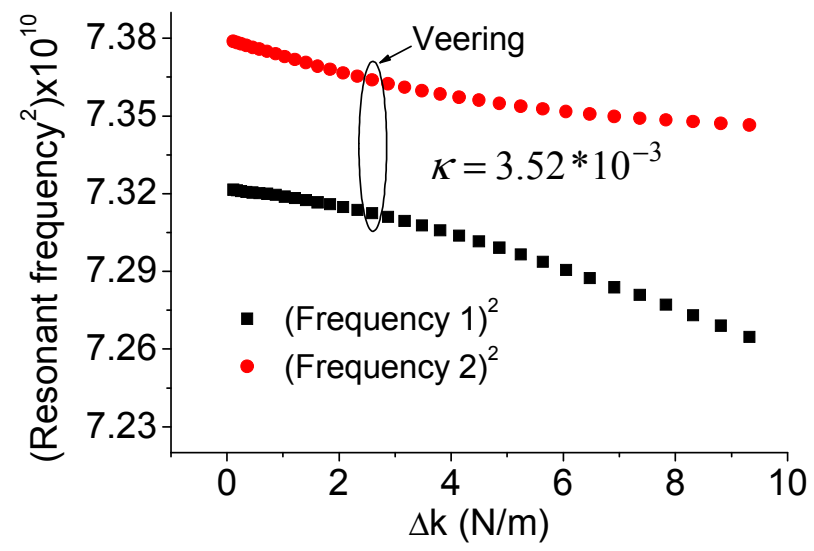

Figure 7: Veering of the loci of the eigenvalues observed when electrically varying the stiffness of resonator 2 .

It may be observed that as the loci of the two eigenvalues approach each other, the eigenvalues do not converge but rather veer away abruptly as predicted by (2). The eigenfunctions at this point of veering hence correspond to those represented in (4) and may be used to directly deduce the scaled coupling factor of the system. The value of $\kappa=k_{c} / k_{1}$ was thus estimated to be equalto $3.52 * 10^{-3}$.

\section{Experiment: Charge sensing}

After estimating the value of $\kappa$, different voltages were applied to the input charge actuation capacitor in order to illustrate the functionality of the device as a mode-localized mechanical charge sensor. Since the capacitance of the charge input port is known, an equivalent input charge may be directly related to an applied voltage. Both the tuning forks were actuated using a DC bias of $7 \mathrm{~V}$ and a source power of
$-20 \mathrm{dBm}$ from the VNA. The S21 parameter response functions of each of the resonators were then measured before and after charge addition and the relative shifts in the eigenstates and those in the eigenvalues were compared at the first mode of vibration. The observed transmission responses measured from the two coupled resonators for different magnitudes of induced charge at the first mode of vibration are represented in Figs. 8 (a) and 8 (b) respectively.
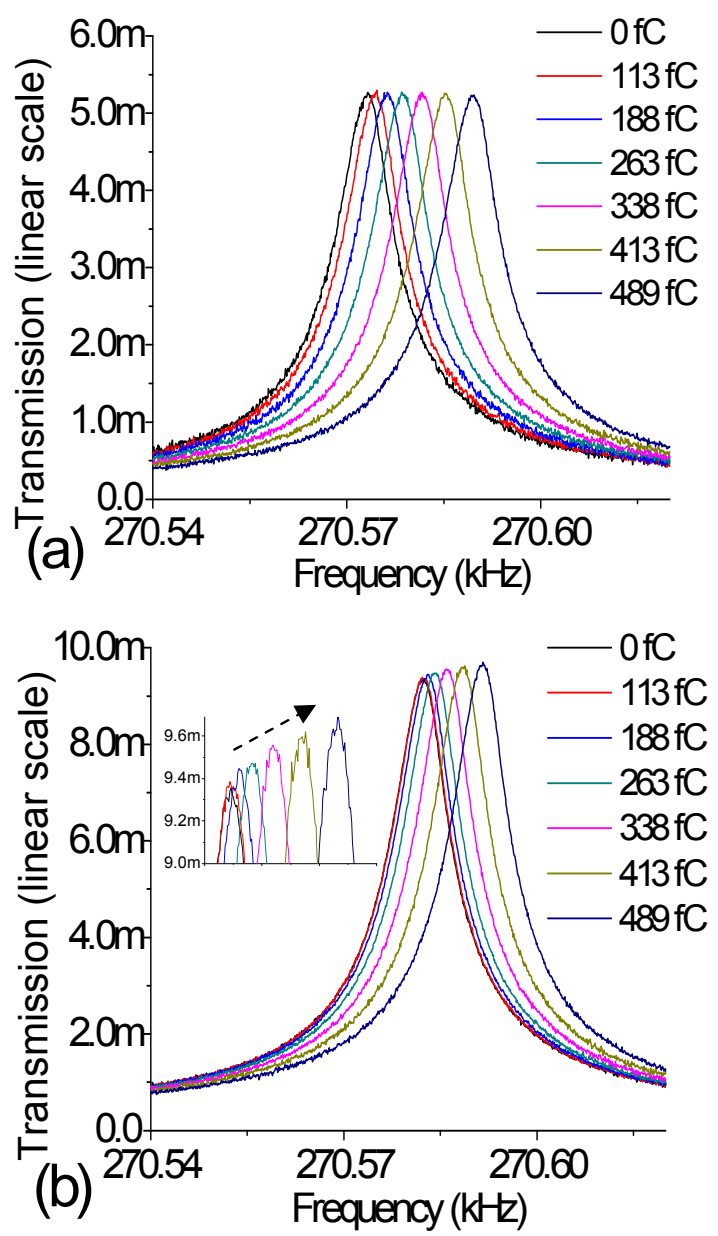

Figure 8: Experimentally observed relative S21 transmission responses measured from ports 1 and 2 for different magnitudes of induced charge at the first fundamental mode of vibration.

From Fig. 8, it may be observed that while the resonant frequency increases with increasing magnitudes of charge input due to the increase in the loaded axial strain in the tines of the tuning fork, the relative amplitudes of vibration at resonance between the resonators also change indicating a localization of the vibration energy within the system. Comparing the relative shifts in the eigenstates with those in the resonant frequency (refer Table 2), it is clear that for the same induced charge addition, the relative shifts in the eigenstates of the system are as high as three orders of magnitude greater than those in resonant frequency as predicted by (11). It is to be noted here that the induced charge may also be translated into an equivalent force / strain as represented in Table 2 thereby widening the relevance of the 
results reported here to force detection, strain monitoring and precision inertial sensing applications as well. Fig. 9 compares the measured eigenstate variations in response to induced charge addition with those predicted using (11).

TABLE II. EXPERIMENTALLY OBSERVED VARIATIONS IN EIGENSTATES FOR DIFFERENT MAGNITUDES OF INDUCED CHARGE INPUT

\begin{tabular}{|l|c|c|c|c|c|}
\hline \multicolumn{6}{|c|}{ Variation in modal dynamics with charge input } \\
\hline \multicolumn{1}{|c|}{ Charge input (fC) } & 0 & 188 & 263 & 413 & 489 \\
\hline Equivalent force input $(\mu \mathrm{N})$ & 0 & 2.3 & 4.6 & 11 & 16 \\
\hline Equivalent strain input $(\mathrm{n} \varepsilon)$ & 0 & 47 & 92 & 228 & 420 \\
\hline Shift in eigenstate (\%) & - & 0.34 & 0.51 & 1.26 & 1.8 \\
\hline $\begin{array}{l}\text { Relative shift in } \\
\text { resonant frequency (\%) }\end{array}$ & - & 0.0011 & 0.0019 & 0.0044 & 0.0060 \\
\hline
\end{tabular}

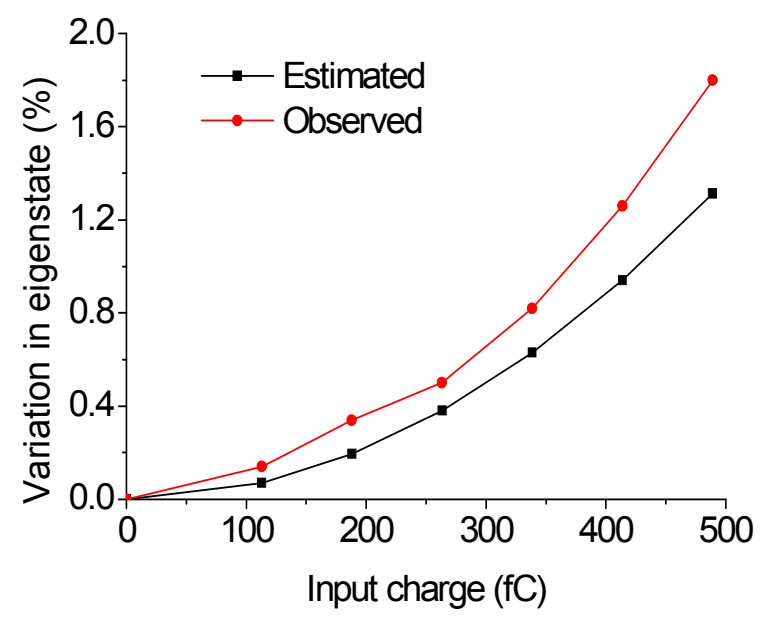

Figure 9: Measured and theoretical variations in the eigenstates for induced charge addition.

\section{CONCLUSION}

In conclusion, this paper presents a novel prototype micromechanical electrometer that utilizes the phenomena of mode localization and curve veering for precise monitoring of charge fluctuations across an input capacitor. Variations in eigenstates that are nearly three orders of magnitude greater than corresponding shifts in the resonant frequency are experimentally demonstrated using a pair of weakly coupled, nearly identical single crystal silicon, double-ended tuning fork (DETF) resonators subjected to differential strain modulation. It is envisaged that further optimization of the device geometry and the incorporation of force leverage mechanisms should also help improve the charge sensitivity of such mode-localized sensors further consequently paving the way for a new class of ultrasensitive mechanical charge sensors.

\section{ACKNOWLEDGMENT}

The authors would like to thank Prof. Jim Woodhouse of the Cambridge University Engineering Department for useful discussions. Mr. P. Thiruvenkatanathan would like to gratefully acknowledge the financial support offered by Churchill College, the British Council and the Cambridge Commonwealth Trust.

\section{REFERENCES}

[1] T. A. Fulton and G. J. Dolan, "Observation of single-electron charging effects in small tunnel junctions," Phys. Rev. Lett., vol. 59, pp. 109112,1987

[2] G. Zimmerli, T. M. Eiles, R. L. Kautz, and J. M. Matinis, "Noise in the Coulomb blockade electrometer," Appl. Phys. Lett., vol. 61, pp. 237239, 1992.

[3] A. N. Cleland and M. L. Roukes, "A nanometer-scale mechanical electrometer," Nature, vol. 392, pp. 160-162, 1998.

[4] P. S. Reihl, K. L. Scott, R. S. Muller, R. T. Howe, and J. A. Yasitis, "Electrostatic charge and field sensors based on micromechanical resonators," J. Mircoelectromech. Syst., vol. 12, pp. 577-589, 2003.

[5] J. E-Y. Lee, Y. Zhu, and A. A. Seshia, "Room temperatire electrometery with sub-10 electron resolution," J. Micromech. Microeng., vol. 18, pp. 025033, 2008.

[6] J. E.-Y. Lee, B. Bahreyni, and A. A. Seshia, "An axial strain modulated double ended tuning fork electrometer," Sens. Actuators A, vol. 148, pp. $395-400,2008$

[7] P. Thiruvenkatanathan, J. Yan, J. Woodhouse, and A. A. Seshia, "Enhancing parametric sensitivity in electrically coupled MEMS resonators," J. Microlectromech. Syst., Vol. 18, pp. 1077-1086, 2009.

[8] C. Pierre, "Mode localization and eigenvalue loci veering phenomena in disordered structures," J. Sound Vib., vol. 126, pp. 485-502, 1988.

[9] C. Pierre and E. H. Dowell, "Localization of vibrations by structural irregularity," J. Sound Vib., vol. 114, pp. 549-564, 1987.

[10] A. W. Leissa, "On a curve veering aberation," J. App. Math. Phys. (ZAMP), vol. 25, pp. 99-111, 1974.

[11] J. R. Kutter and V. G. Sigillito, “On curve veering," J. Sound Vib., vol. 75 , pp. $585-588,1981$.

[12] N. C. Perkins and C. D. Mote Jr., "Comments on curve veering in eigenvalue problems," J. Sound Vib., vol. 106, pp. 451 - 463, 1986.

[13] X. L. Liu, "Behaviour of derivative of eigenvalues and eigenvectors in curve veering and mode localization and their relation to close eigenvalues," J. Sound Vib., vol. 256, pp. 551-564, 2002.

[14] A. A. Seshia, M. Palaniapan, T. A. Roessig, R. T. Howe, R. W. Gooch, T. R. Schimert, and S. Montague, "A Vacuum Packaged Surface Micromachined Resonant Accelerometer," J. Microelectromech. Syst., vol. 11, pp. $784-793,2002$.

[15] S. X. P. Su, H. S. Yang, and A. M. Agogino, "A resonant accelerometer with two-stage microleverage mechanisms fabricated by SOI-MEMS technology,” IEEE Sens. J., vol. 5, pp. 1214-1222, 2005. 